\title{
Single-Chirality Near-Infrared Carbon Nanotube Sub-Cellular Imaging and FRET Probes
}

Rachel Langenbacher ${ }^{\dagger}$, Januka Budhathoki-Upretyo ${ }^{\circ}$, Prakrit V Jena ${ }^{\ddagger}$, Daniel Roxbury ${ }^{\S}$, Jason Streit ${ }^{\nabla}$, Ming Zheng ${ }^{\diamond}$, Daniel A. Heller ${ }^{\dagger * *}$

$\dagger$ Weill Cornell Medical College, New York, New York 10065, United States

o Department of Textile Engineering, Chemistry, and Science, North Carolina State University, Raleigh, North Carolina 27695, United States

$\$$ Memorial Sloan Kettering Cancer Center, New York, New York 10065, United States

$\S$ Department of Chemical Engineering, University of Rhode Island, Kingston, Rhode Island 02881, United States

- Air Force Research Laboratory, Wright-Patterson Air Force Base Ohio, 45433, United States

${ }^{\diamond}$ National Institute of Standards and Technology, Gaithersburg Maryland, 20089, United States 
A.

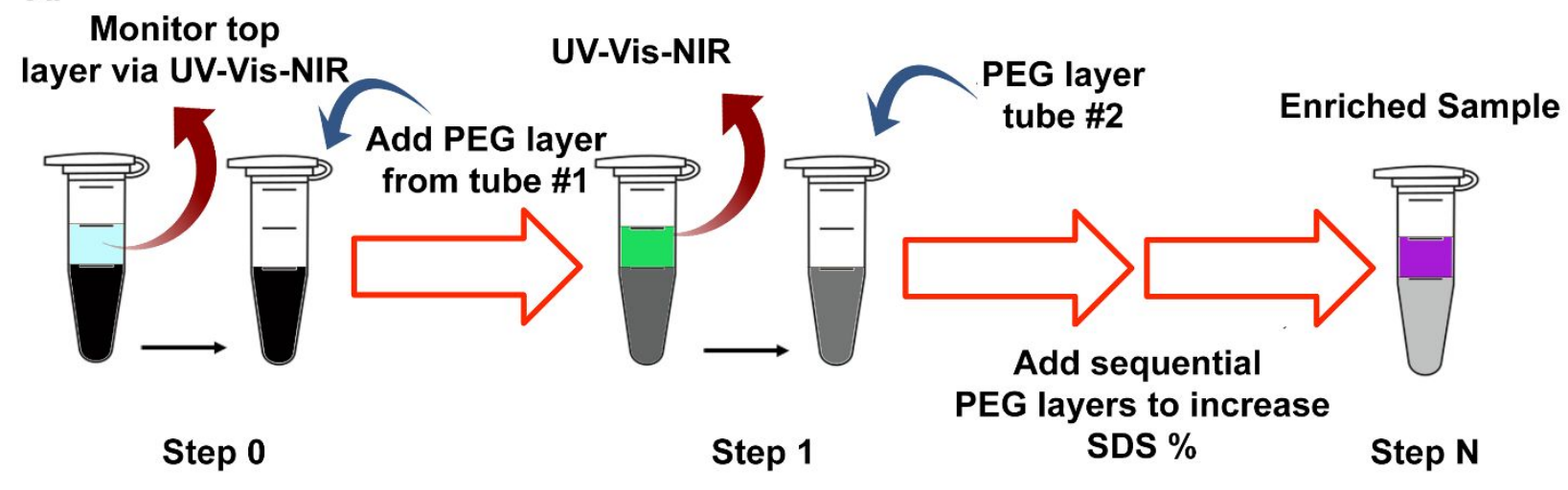

B.

c.

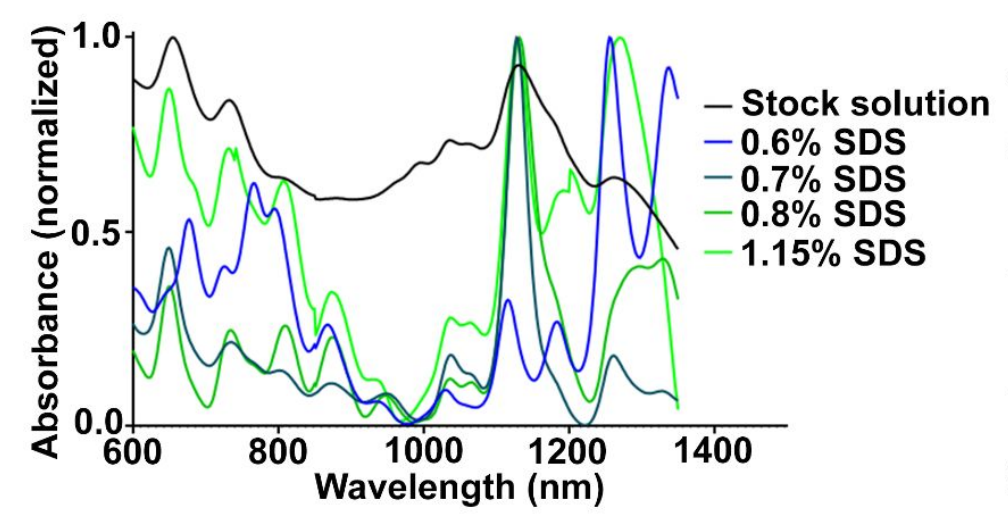

D.
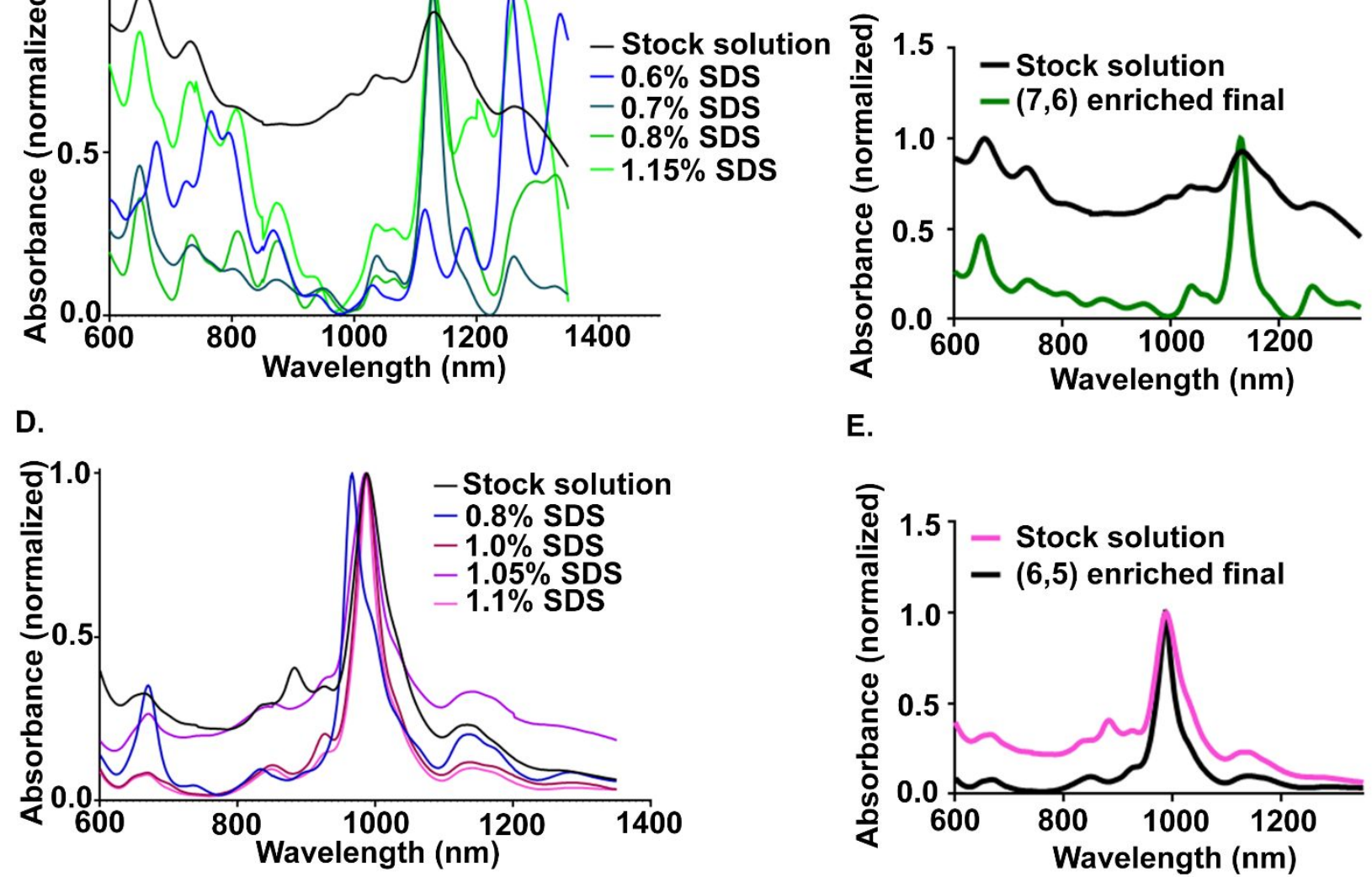

E.

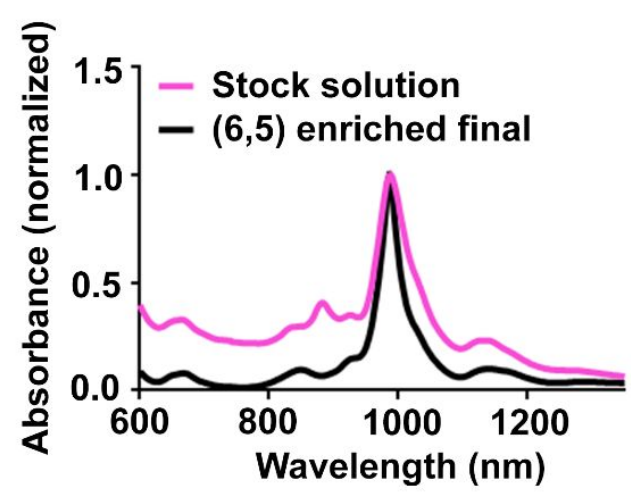

Figure S1. ATPE separation of single-walled carbon nanotubes. (A) Scheme of the ATPE separation method. (B) Absorbance spectra of representative top fractions from the ATPE separation of the $(7,6)$ SWCNT chirality, at different SDS concentrations with DOC held constant. (C) Representative spectra of the initial stock suspension and the final fraction of the $(7,6)$ enrichment protocol. (D) Absorbance spectra of representative top fractions from the ATPE separation of the $(6,5)$ chirality, at different SDS concentrations with DOC held constant. (E) 
Representative spectra of the initial stock suspension and the final fraction of the $(6,5)$ enrichment protocol. All spectra normalized to maxima. 


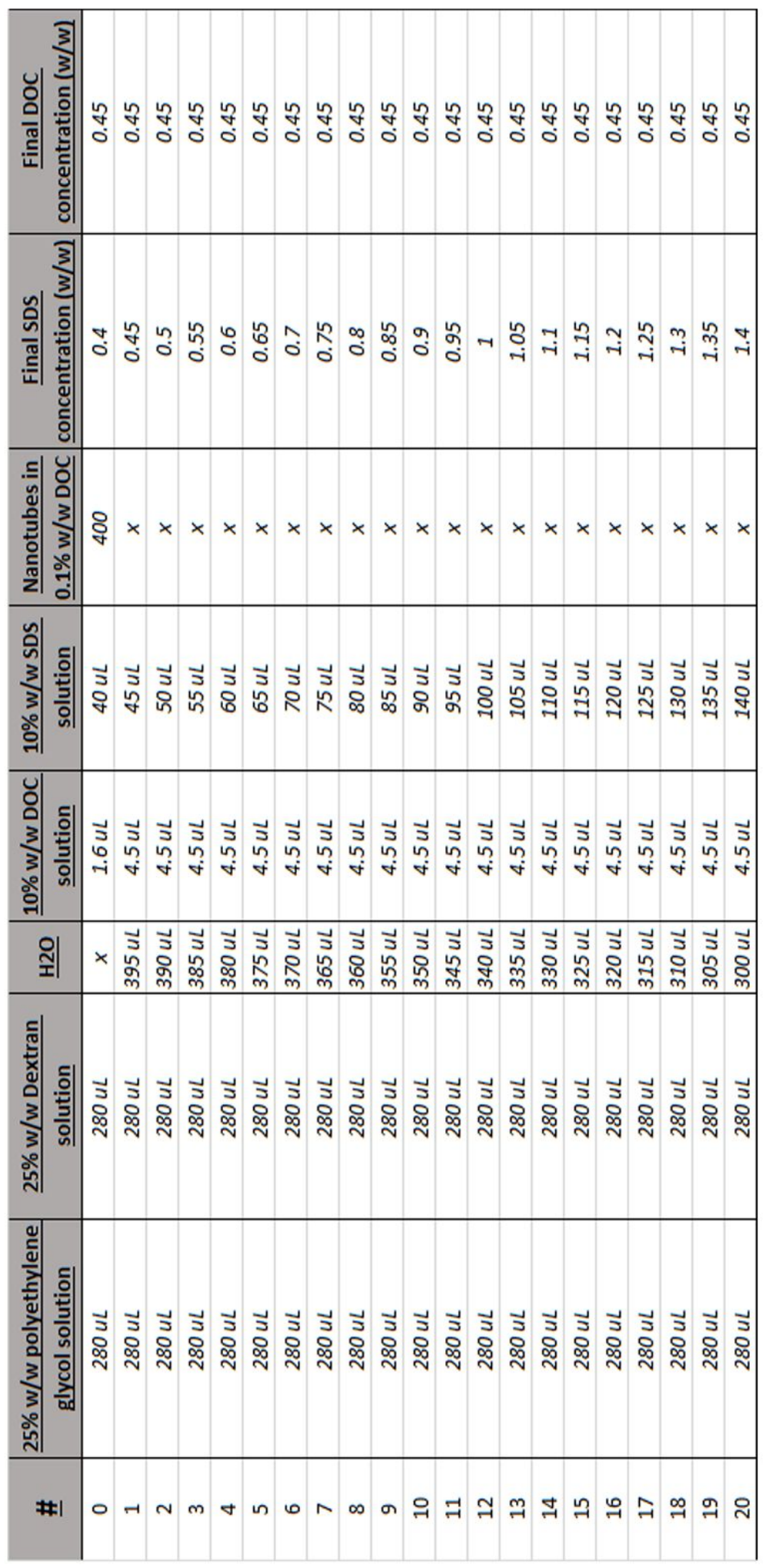

Table S1. ATPE separation conditions. Volumes and concentrations of reagents used in the ATPE protocol. 
A.

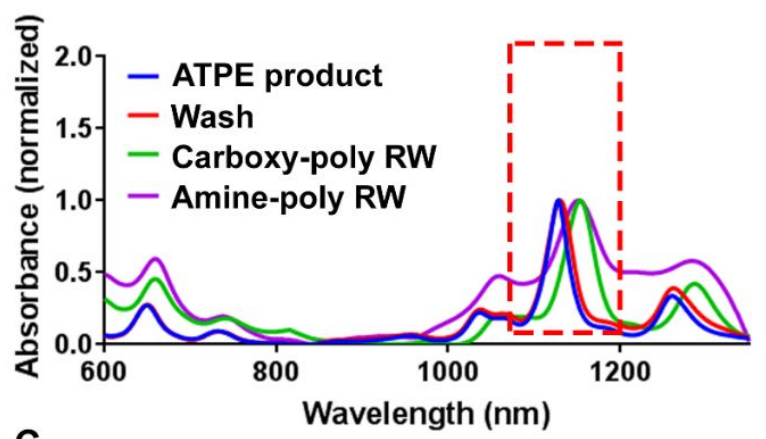

C.

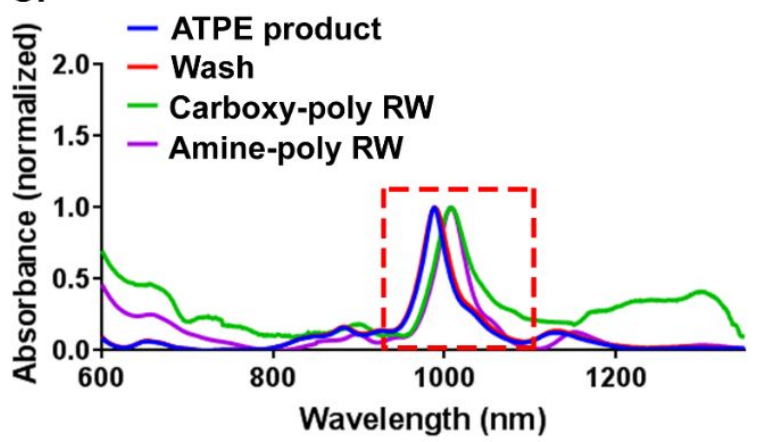

B.

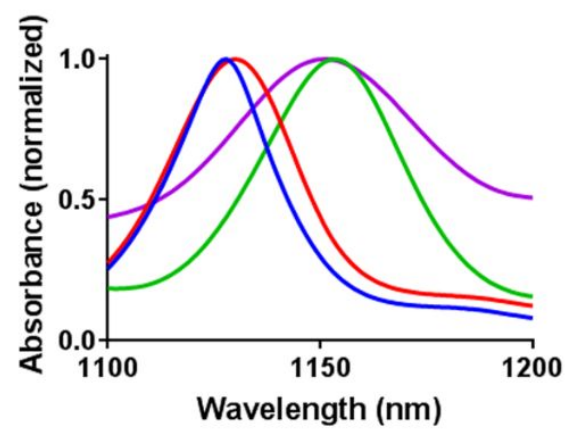

D.

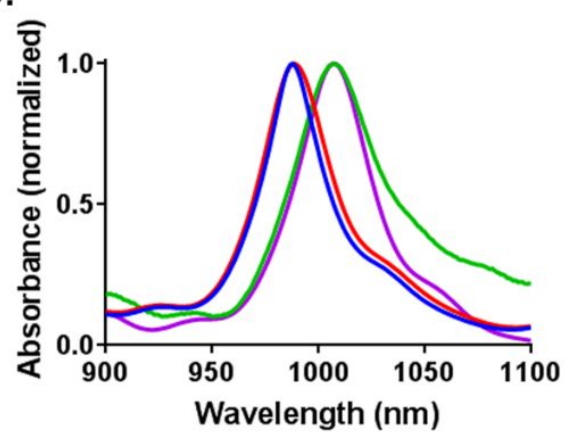

Figure S2. Absorbance spectra of rewrapped nanotubes. (A) Normalized absorbance spectra of $(7,6)$ ATPE product stabilized in DOC (blue line), sorted nanotubes after one wash prior to polycarbodiimide addition (red line), final rewrapping (RW) product carboxy-poly-(7,6) (green line), and final rewrapping product $(7,6)$ amine-poly. (B) Expanded section of absorbance spectra indicated by red dotted box in (A). (C) Normalized absorbance spectra of $(6,5)$ ATPE product stabilized in surfactant (blue line), sorted nanotubes after one wash prior to polycarbodiimide addition (red line), final rewrapping product $(6,5)$ carboxy-poly (green line), and final rewrapping product $(6,5)$ amine-poly. (D) Expanded section of absorbance spectra indicated by red dotted box in $(\mathrm{C})$. All spectra normalized to maxima. 


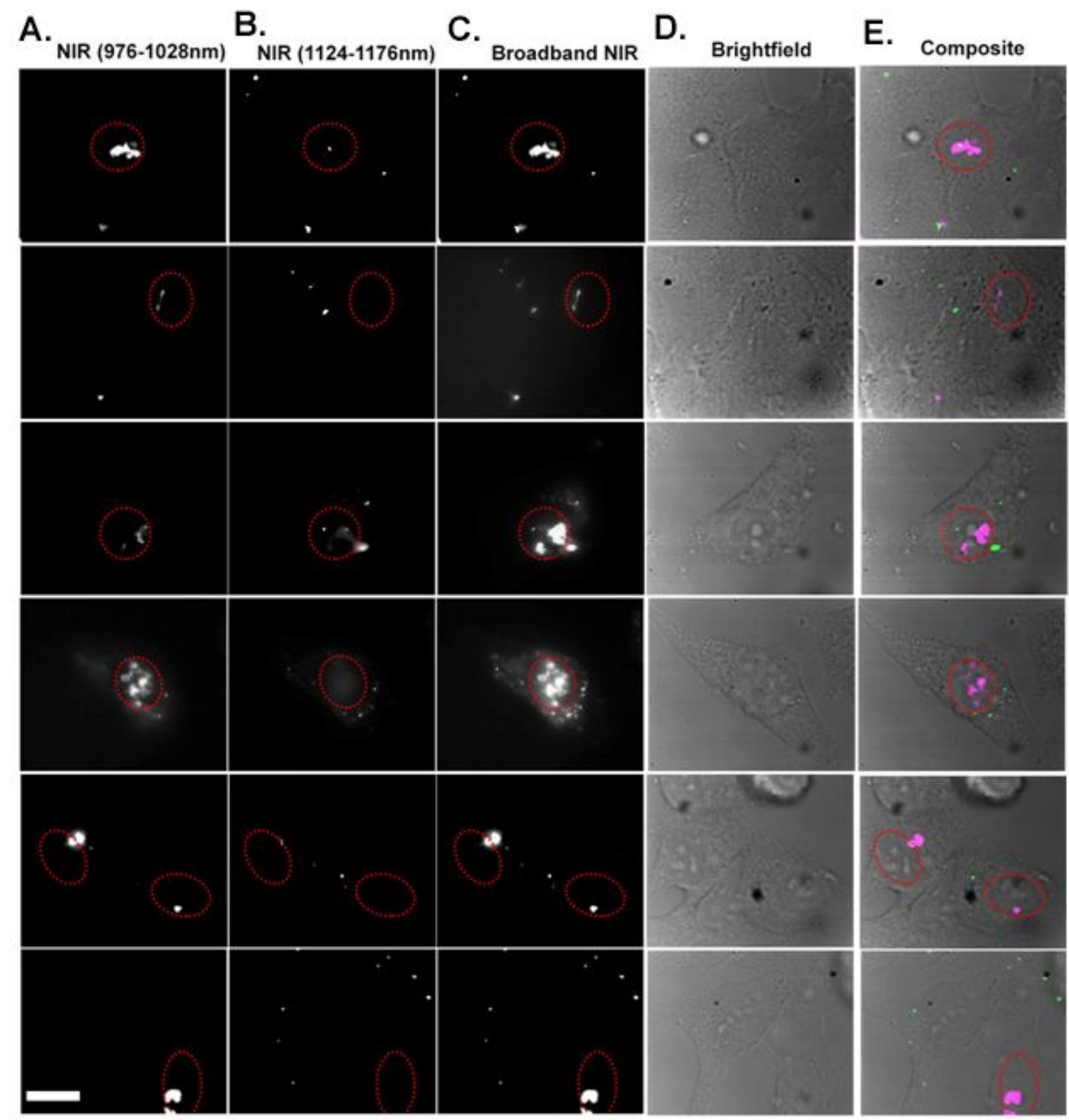

Figure S3: Representative images of HeLa cells treated with $(7,6)$ amine-poly SWCNTs and $(6,5)$ guanidinium-poly SWCNTs (A) NIR emission from $976 \mathrm{~nm}-1028 \mathrm{~nm}$. (B) NIR emission from 1124 nm-1175 nm. (C) Broadband NIR. (D) Brightfield. (E) Composite. Scalebar $=10 \mu \mathrm{m}$. 
A.

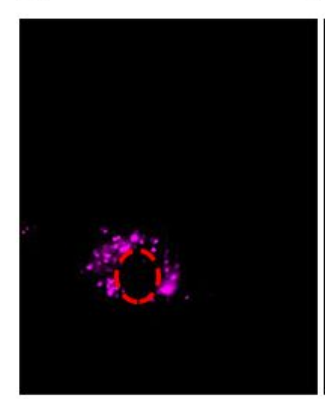

NIR (976-1028nm)

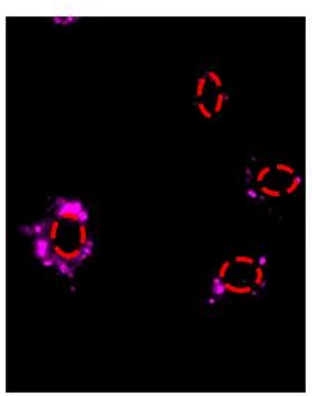

B.

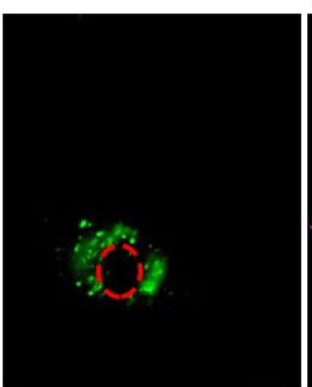

NIR (1124-1176)

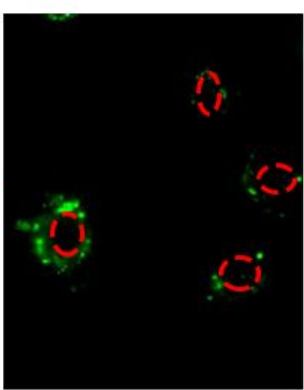

C.

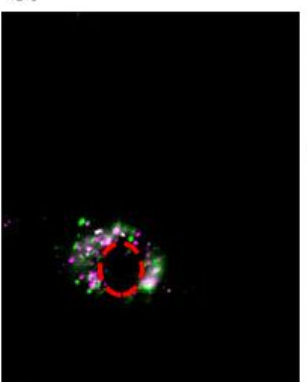

Composite NIR

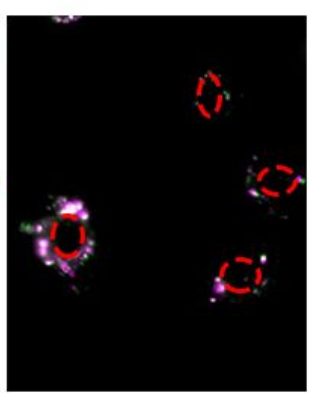

D.

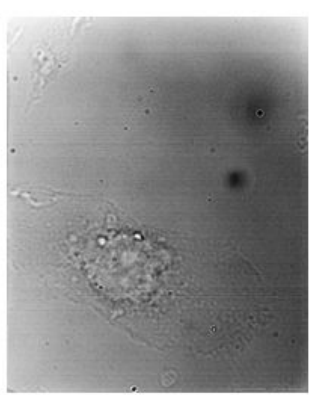

Brightfield

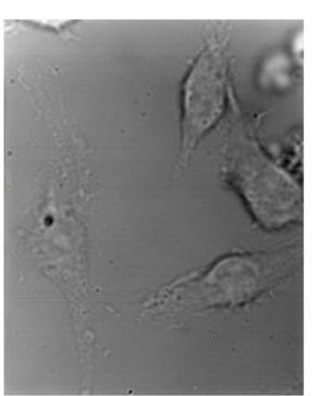

E.

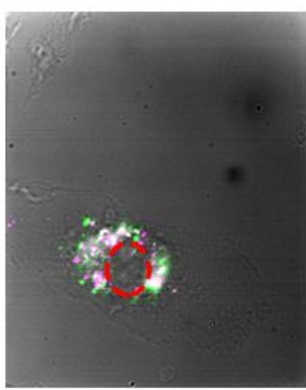

Composite

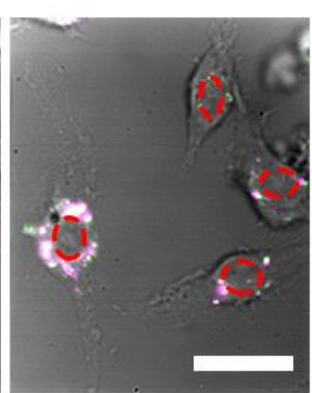

Figure S4: Representative images of HeLa cells treated with $(7,6)$ amine-poly SWCNTs and $(6,5)$ amine-poly SWCNTs (A) NIR emission from $976 \mathrm{~nm}-1028 \mathrm{~nm}$. (B) NIR emission from 1124 nm-1175 nm. (C) NIR composite. (D) Brightfield. (E) Composite. Scalebar $=10 \mu \mathrm{m}$. 


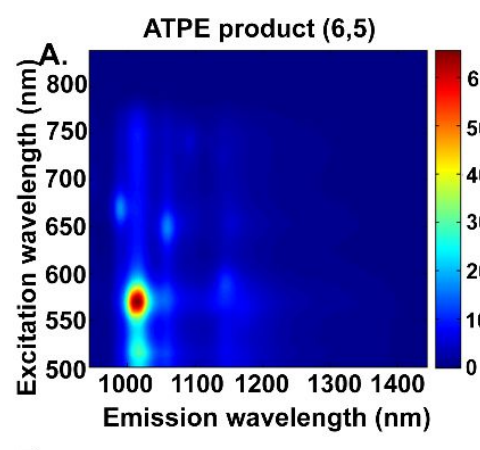

D.

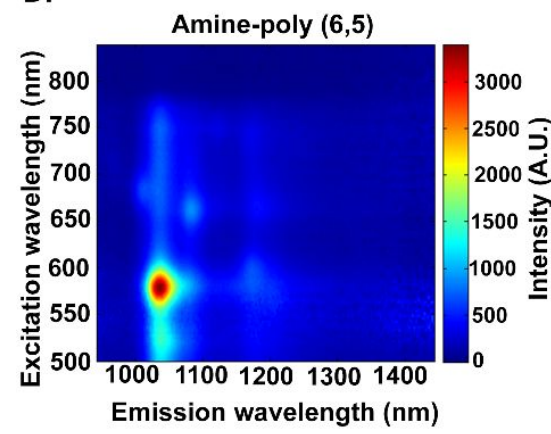

F.

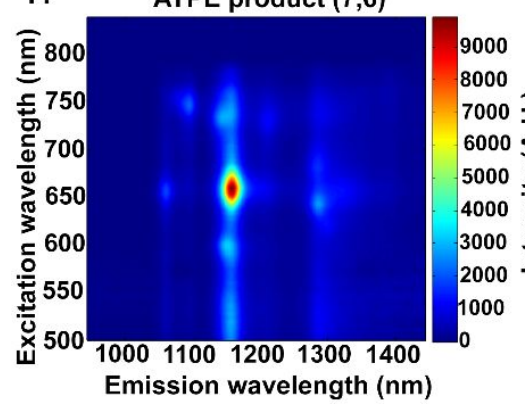

I.

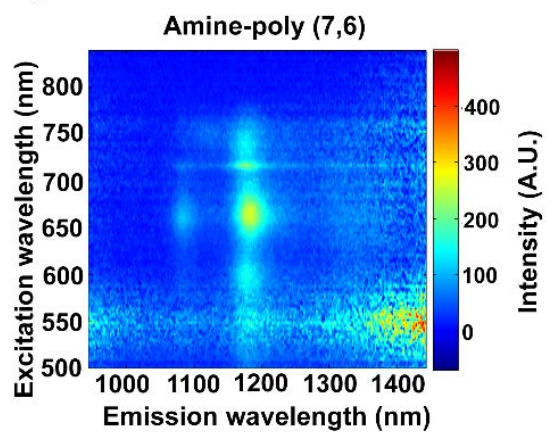

B.

B.

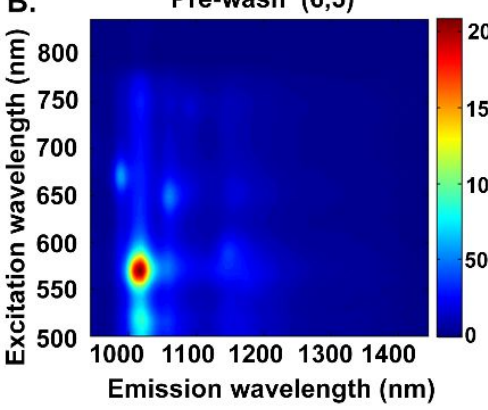

E.

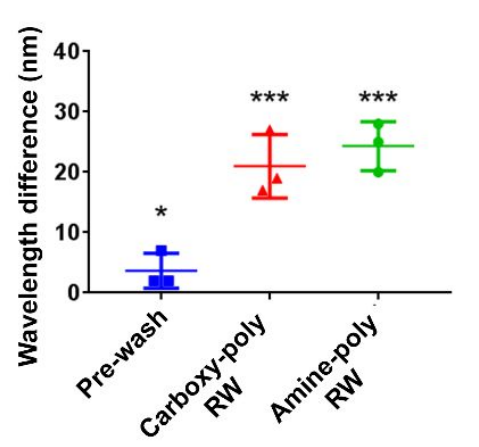

C.

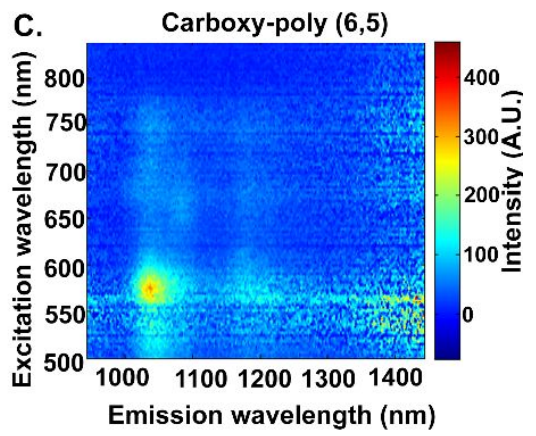

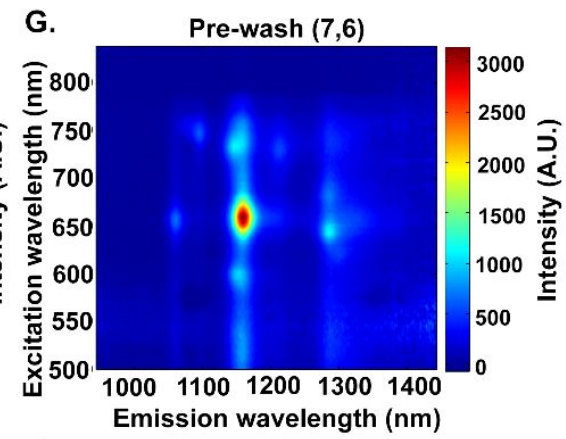

J.
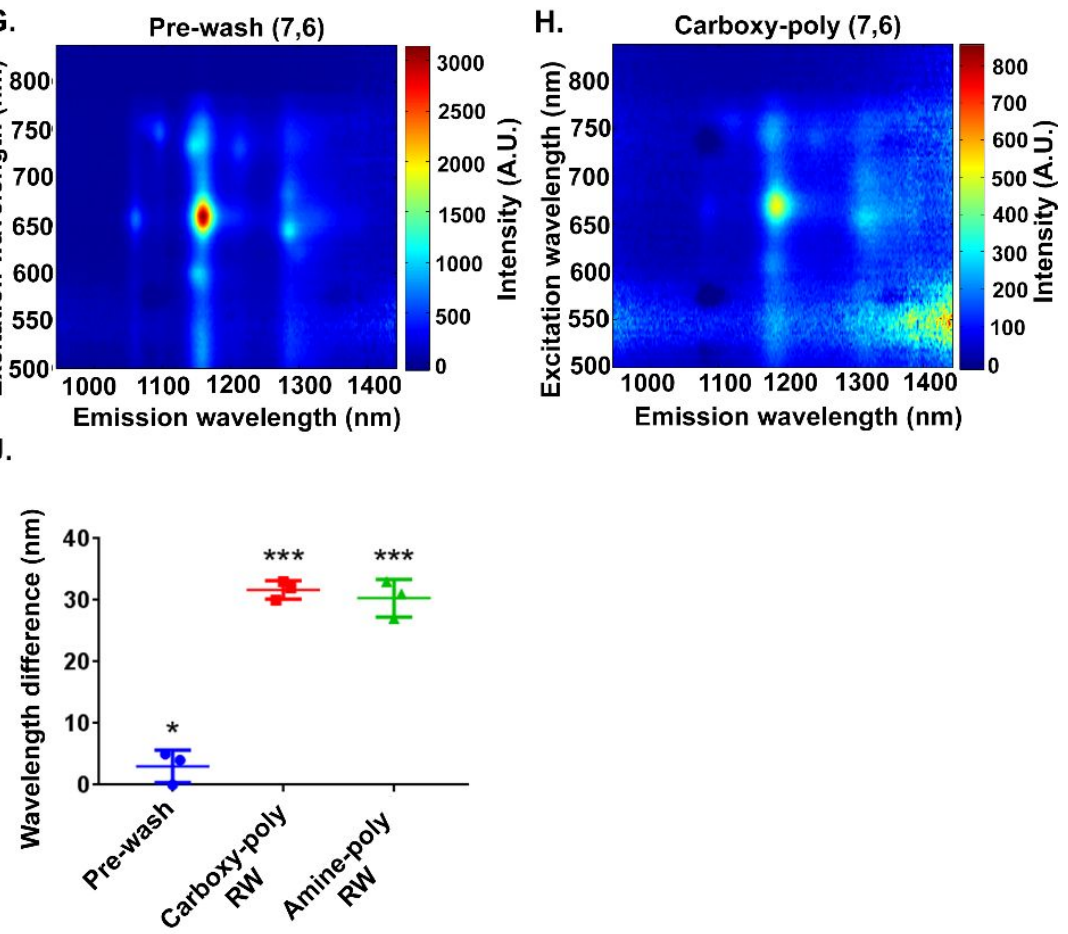

Figure S5. Representative photoluminescence excitation-emission plots of ATPE-separated and rewrapped samples. Representative PL spectra of: (A) ATPE product $(6,5)$ in $0.1 \%$ DOC, (B) $(6,5)$ sorted nanotubes after one wash prior to polycarbodiimide addition, and (C) $(6,5)$ carboxy-poly rewrapped product. (D) (6,5) amine-poly rewrapped product. (E) Emission wavelength shift of the $(6,5)$ species normalized to ATPE product emission. Representative PL 
spectra of: (F) ATPE product $(7,6)$ in surfactant. $(G)(7,6)$ sorted nanotubes after one wash prior to polycarbodiimide addition, $(\mathrm{H})(7,6)$ carboxy-poly rewrapped product, and (I) $(7,6)$ amine-poly rewrapped product. (J) Emission wavelength shift of the $(7,6)$ species normalized to ATPE product emission. 